\title{
Tratamento Percutâneo de Lesões Residuais e Complicações em Condutos de Pacientes Submetidos a Cirurgia de Circuito do Tipo Fontan
}

\author{
João Luiz Manica', André Luis Bodini' ${ }^{1}$, Paulo Renato Machado', \\ Mônica Scott Borges ${ }^{1}$, Raul Ivo Rossi Filho'
}

\section{RESUMO}

Introdução: A introdução da cirurgia de anastomose cavopulmonar total (ACPT) ou cirurgia de Fontan modificou de forma significativa a história natural de pacientes com cardiopatias complexas não passíveis de reparo biventricular. Entretanto, é conhecido o desenvolvimento de estenoses silenciosas. Além disso, a utilização de condutos fenestrados é uma técnica comumente realizada. O presente estudo relata a experiência de um centro terciário no implante percutâneo de dispositivos em condutos de pacientes com circulação do tipo univentricular. Método: Entre julho de 2000 e julho de 2010, 12 pacientes receberam dispositivos percutâneos em condutos após cirurgia de Fontan. Os pacientes foram divididos em dois grupos, de acordo com a indicação para o procedimento: 5 pacientes receberam implante de oclusores septais para fechamento de fenestração cirúrgica (grupo 1), 6 pacientes receberam stents para alívio de obstrução de condutos (grupo 2), e 1 paciente recebeu ambos os dispositivos simultaneamente. A média de idade dos pacientes do grupo 1 no momento do procedimento era de $174+53,5$ meses e o peso médio era de $30,7 \pm 6,8 \mathrm{~kg}$. A média de idade dos pacientes do grupo 2 no momento do procedimento era de $148,5 \pm 84,6$ meses e o peso médio era de $28,9+19,8 \mathrm{~kg}$. Resultados: No grupo 1 , a saturação média passou de $82,6 \pm 7,5 \%$ para $90,4+7,5 \%$ logo após o procedimento $(P=0,001)$. No grupo 2 , a saturação média passou de $81,8 \pm 8,9 \%$ para $91,3 \pm 8,7 \%(P=0,01)$. O menor diâmetro do conduto passou de uma média de $6,9 \pm 4,8 \mathrm{~mm}$ para $16,6 \pm 3,5 \mathrm{~mm}$ após o procedimento $(P=0,02)$. O paciente que realizou ambos os procedimentos simultaneamente teve aumento do menor diâmetro do conduto de $11,7 \mathrm{~mm}$ para $16 \mathrm{~mm}$ e melhora da saturação sistêmica, passando de $60 \%$ para $90 \%$. Conclusão: O tratamento da obstrução de condutos por meio do implante de stents não-recobertos e a oclusão

\author{
ABSTRACT
Percutaneous Treatment of Residual Lesions and Complications in Conduits Following Fontan Procedure

Background: The introduction of total cavopulmonary anastomosis (TCPA) or Fontan procedure significantly changed the natural history of patients with complex heart diseases inadequate for biventricular repair. However, the development of silent stenosis is well known. In addition, fenestrated conduits are frequently used. The present study reports the experience of a tertiary center with percutaneous devices implanted in the conduits of patients with univentricular circulation. Method: From July 2000 to July 2010, 12 patients received percutaneous devices in conduits after Fontan procedure. Patients were divided into two groups, according to the indication for the procedure: 5 patients received septal occluders to close surgical fenestrations (group 1), 6 patients received stents for the relief of conduit obstructions (group 2) and 1 patient received both devices simultaneously. Mean age of group 1 patients at the time of the procedure was $174 \pm 53.5$ months and mean weight was $30.7+6.8 \mathrm{~kg}$. Mean age of group 2 patients was $148.5 \pm 84.6$ months and mean weight was $28.9 \pm 19.8 \mathrm{~kg}$. Results: In group 1, oxygen saturation rose from $82.6+7.5 \%$ to $90.4+7.5 \%$ immediately after the procedure $(\mathrm{P}=0.001)$. In group 2 , oxygen saturation rose from $81.8+8.9 \%$ to $91.3+8.7 \%(P=0.01)$. The minimal conduit diameter changed from $6.9+4,8 \mathrm{~mm}$ to $16.6 \pm$ $3.5 \mathrm{~mm}$ after the procedure $(P=0.02)$. The patient who was submitted to both procedures simultaneously had an increase of the minimal conduit diameter from $11.7 \mathrm{~mm}$ to $16 \mathrm{~mm}$ and an improvement in systemic saturation from $60 \%$ to $90 \%$. Conclusion: Treatment of conduit obstructions with non-covered stents and the occlusion of surgical

1 Serviço de Cardiologia Intervencionista em Cardiopatias Congênitas do Instituto de Cardiologia do Rio Grande do Sul - Fundação Universitária de Cardiologia (IC-FUC) - Porto Alegre, RS, Brasil. Correspondência: Raul Ivo Rossi Filho. Av. Princesa Isabel, 370 Santana - Porto Alegre, RS, Brasil - CEP 90620-001

E-mail: rrossi.voy@terra.com.br

Recebido em: 27/9/2010 • Aceito em: 28/11/2010 
Manica JL, et al. Tratamento Percutâneo de Lesões Residuais e Complicações em Condutos de Pacientes Submetidos a Cirurgia de Circuito do Tipo Fontan. Rev Bras Cardiol Invasiva. 2010;18(4):435-42.

de fenestrações cirúrgicas com stents recobertos ou dispositivos de Amplatzer ${ }^{\mathrm{TM}}$ são procedimentos seguros, com altos índices de sucesso imediato e que se mantêm a médio prazo.

DESCRITORES: Stents. Cardiopatias congênitas. Próteses e implantes. fenestrations with covered stents or Amplatzer ${ }^{\mathrm{TM}}$ devices are safe procedures, with high immediate success rates which are maintained in the mid-term follow-up.

KEY-WORDS: Stents. Heart defects, congenital. Prostheses and implants.
$\mathrm{H}$ istoricamente, pacientes com cardiopatias complexas, não passíveis de reparo biventricular, eram encaminhados para cirurgias paliativas com mortalidade precoce e pobre qualidade de vida em decorrência de dessaturação constante e de progressiva disfunção da câmara principal. A introdução da cirurgia de anastomose cavopulmonar total ou cirurgia de Fontan, inicialmente por meio de anastomose atriopulmonar, alterou de forma significativa a história natural desses pacientes. ${ }^{1}$ Entretanto, a alta incidência de arritmias decorrentes de dilatação atrial, atriotomia e linhas de sutura atriais levou à criação da anastomose através de conduto extracardíaco, que, dentre outras vantagens, diminuiu drasticamente a incidência de arritmias a longo prazo. A incidência de estenose de condutos intra ou extracardíacos, ainda que baixa, não é irrelevante, visto que esses pacientes são submetidos ao procedimento cirúrgico ainda crianças e o crescimento somático pode acarretar torção do conduto. ${ }^{2}$ Além disso, a utilização de condutos fenestrados é uma técnica ainda comumente utilizada, que permite melhor adaptação pós-operatória a despeito de discreta cianose. ${ }^{3}$ Segundo diversos estudos, o uso de dispositivos percutâneos capazes de ocluir fenestrações é bastante seguro e eficaz, além de prevenir novo procedimento cirúrgico em pacientes já submetidos a cirurgias prévias. ${ }^{4-6}$ Entretanto, existem poucos relatos de utilização de stents para alívio de estenoses em condutos intra ou extracardíacos de pacientes submetidos a cirurgia de anastomose cavopulmonar total. ${ }^{7} \mathrm{O}$ presente estudo relata a experiência de um centro terciário no implante percutâneo de dispositivos em condutos de pacientes com circulação do tipo univentricular.

\section{MÉTODO}

\section{População do estudo}

O estudo compreende uma coorte retrospectiva de todos os pacientes em pós-operatório de anastomose cavopulmonar total com conduto intra ou extracardíaco, submetidos a implante de stents para alívio de obstrução de conduto e/ou dispositivos para oclusão de fenestração em nossa instituição.

De julho de 2000 a julho de 2010, 12 pacientes receberam dispositivos percutâneos em condutos após cirurgia de Fontan. Os pacientes foram divididos em dois grupos, de acordo com a indicação para o procedimento: cianose importante decorrente de fenestração cirúrgica (grupo 1) e sinais clínicos ou ecocardiográficos de obstrução do conduto (grupo 2), e um paciente foi submetido a ambos os procedimentos simultaneamente por apresentar sinais de obstrução do conduto associado a fenestração cirúrgica.

O grupo 1 incluiu 5 pacientes cianóticos portadores de fenestrações cirúrgicas, 2 dos quais receberam stents recobertos e 3 receberam oclusores septais Amplatzer $^{\text {TM }}$ (AGA, Golden Valley, Estados Unidos) para a oclusão das fenestrações. O grupo 2 compreendeu 6 pacientes com evidências de obstrução do conduto cavopulmonar e congestão sistêmica, 2 dos quais receberam stents recobertos e 4 receberam stents não-recobertos para alívio da obstrução. Dentre os pacientes que receberam stents recobertos, um deles apresentava quadro de trombose maciça do tubo de dácron extracardíaco (paciente 10). Havia obstrução completa do conduto imediatamente acima das veias hepáticas e congestão sistêmica refratária ao tratamento clínico, evidenciando a necessidade de intervenção (Figura 1). O outro paciente apresentava retração importante de conduto extracardíaco calcificado com abundante circulação colateral venosa intra-hepática para átrio direito (paciente 11).

Além disso, um paciente com quadro de cansaço aos médios esforços e cianose, já com stent previamente implantado em ramo pulmonar esquerdo, realizou oclusão de fenestração cirúrgica com dispositivo de Amplatzer ${ }^{\circledR}$ simultaneamente ao alívio de obstrução de conduto intracardíaco com stent não-recoberto ( $\mathrm{Fi}$ gura 2).

Os dados demográficos dos dois grupos do estudo estão apresentados nas Tabelas 1 e 2 .

\section{Procedimento e técnica}

\section{Oclusão de fenestrações}

Acesso femoral foi obtido para avaliação hemodinâmica e angiográfica completa do circuito de Fontan. Saturações venosas sistêmicas, bem como as pressões, foram obtidas em todo o circuito. Angiografias com cateter Berman ${ }^{\mathrm{TM}}$ (Critikon Inc., Estados Unidos) foram realizadas em veias cavas superior e inferior e artérias pulmonares para avaliar a morfologia do circuito 

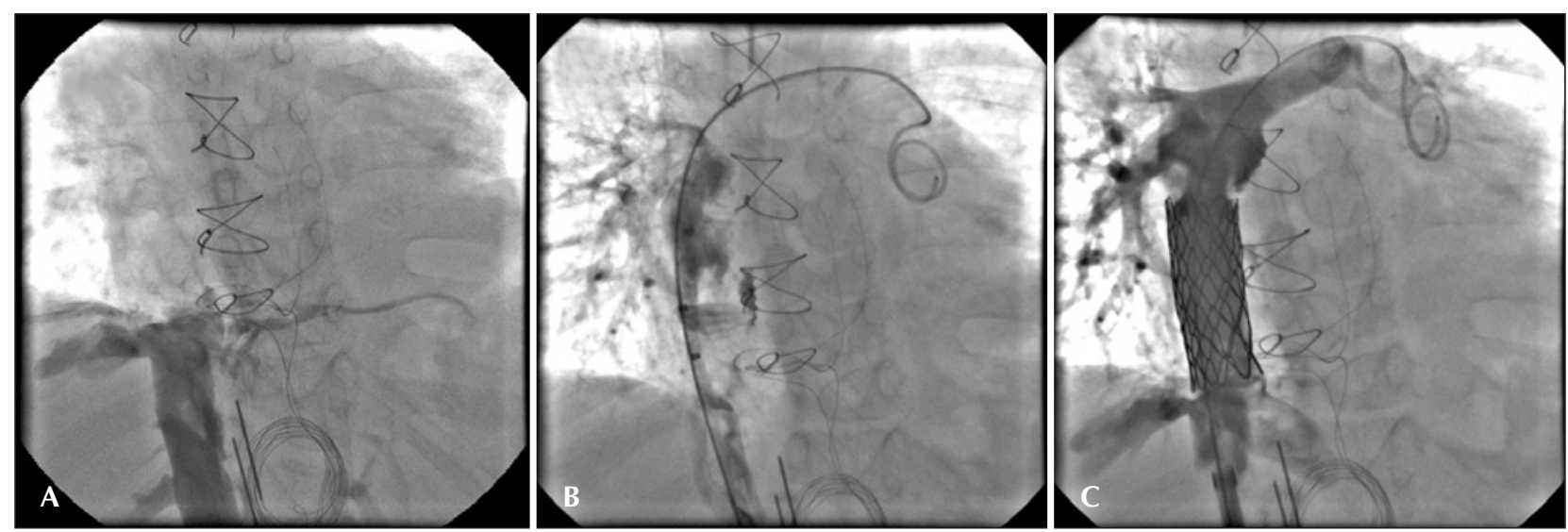

Figura 1 - Stent recoberto para obstrução total de conduto extracardíaco com tubo de dácron. Em A, angiografia com cateter pigtail $6 \mathrm{~F}$ na anastomose da veia cava inferior, demonstrando obstrução completa do fluxo no conduto imediatamente acima das veias hepáticas. Em B, passagem através do conduto com guia extrarrígida, demonstrando perviedade desse conduto. A injeção com cateter MultiTrack evidenciou extensa formação trombótica no interior do conduto. Em C, injeção final em bainha longa após implante de stent recoberto.
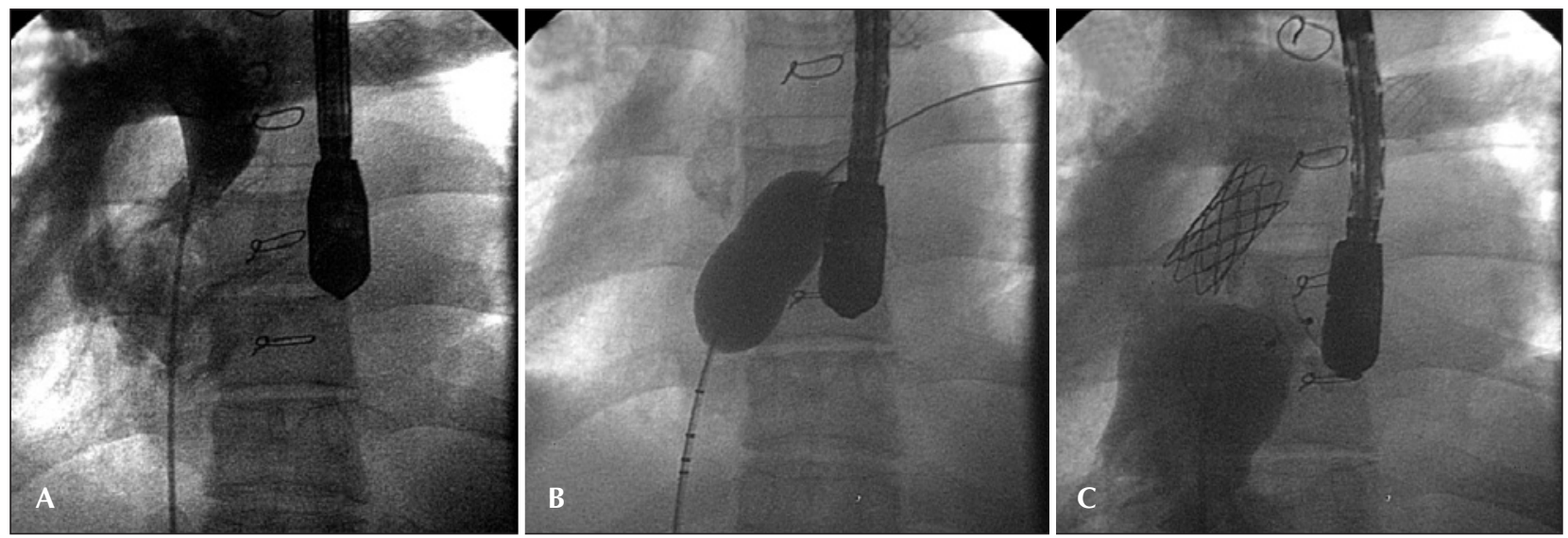

Figura 2 - Implante de stent em estenose de conduto intracardíaco e oclusão percutânea de fenestração cirúrgica. Em A, angiografia com cateter pigtail, demonstrando retração do conduto intracardíaco e presença de escape de fluxo através da fenestração de difícil delimitação, além de stent previamente implantado em ramo pulmonar esquerdo com adequado resultado. Em B, balão de medição através da fenestração para adequada delimitação e realização de teste de oclusão do defeito. É possível visualizar a presença de calcificação na região da estenose do conduto. Em C, angiografia de controle após liberação do stent e da prótese de Amplatzer ${ }^{\top M}$, demonstrando adequado fluxo anterógrado sem shunt residual através da fenestração.

de Fontan e para definir a localização da fenestração cirúrgica além de possíveis vasos venosos colaterais. Após cruzar a fenestração, guia extrarrígida foi posicionada nas veias pulmonares ou na cavidade atrial, através da qual balão de medição foi insuflado para teste de oclusão da fenestração com monitorização de saturação arterial de oxigênio bem como de pressão arterial sistêmica e de enchimento. Na ausência de aumento significativo da pressão venosa sistêmica, queda da saturação de oxigênio ou sinais de baixo débito cardíaco, deu-se prosseguimento à oclusão da fenestração com prótese de Amplatzer ${ }^{\mathrm{TM}}$.

\section{Implante de stents em condutos}

Acesso femoral foi obtido em 6 pacientes e acesso jugular em 2 pacientes por obstrução venosa femoral crônica. Após criterioso estudo manométrico e ana- tômico realizado conforme descrito previamente para oclusão de fenestrações, bainha longa foi introduzida através do conduto sobre guia extrarrígida na qual foi avançado o stent montado manualmente em balão com diâmetro previamente escolhido e insuflado para desobstrução do conduto e/ou oclusão de fenestração, no caso de stents recobertos. Todos os pacientes receberam heparina $(100 \mathrm{UI} / \mathrm{kg})$ e profilaxia com antibióticos endovenosos.

Todos os casos foram realizados sob anestesia geral, de acordo com o protocolo da instituição. Consentimento informado foi obtido para todos os procedimentos.

\section{Seguimento}

Os pacientes foram seguidos no ambulatório de cardiologia pediátrica da instituição e os dados clínicos 
TABELA 1

Dados demográficos do grupo 1

\begin{tabular}{|c|c|c|c|c|c|c|}
\hline & $\begin{array}{l}\text { Idade } \\
\text { (meses) }\end{array}$ & $\begin{array}{c}\text { Peso } \\
\text { (kg) }\end{array}$ & Diagnóstico & Conduto & $\begin{array}{l}\text { Tempo após } \\
\text { Fontan (meses) }\end{array}$ & Dispositivos \\
\hline 1 & 157 & 35 & DVEVE/TGA/CIV & $\begin{array}{l}\text { Homoenxerto } \\
\text { aórtico com leak }\end{array}$ & 65 & $\begin{array}{c}\text { CP Covered } 45 \text { mm } \\
\text { Balão } 16 \text { mm e } 20 \text { mm }\end{array}$ \\
\hline 2 & 189 & 25 & $\begin{array}{l}\text { Anomalia } \\
\text { de Ebstein }\end{array}$ & $\begin{array}{l}\text { Intracardíaco } \\
\text { fenestrado }\end{array}$ & 120 & $\begin{array}{c}\text { ADVANTA } \\
\text { Covered V12 } 16 \mathrm{~mm} \times 4 \mathrm{~mm}\end{array}$ \\
\hline 3 & 235 & 30 & DVEVE/TGA/CIV/PCA & $\begin{array}{l}\text { Intracardíaco } \\
\text { fenestrado }\end{array}$ & 65 & $\begin{array}{c}\text { Amplatzer ASO } \\
12 \mathrm{~mm}\end{array}$ \\
\hline 4 & 93 & 23,5 & $\begin{array}{l}\text { Atresia pulmonar } \\
\text { com SIV íntegro }\end{array}$ & $\begin{array}{l}\text { Intracardíaco } \\
\text { fenestrado }\end{array}$ & 60 & $\begin{array}{l}\text { Amplatzer CRIBI } \\
18 \mathrm{~mm}\end{array}$ \\
\hline 5 & 200 & 40 & $\begin{array}{c}\text { DVEVD } \\
\text { DVSVD/EP }\end{array}$ & $\begin{array}{l}\text { Intracardíaco } \\
\text { fenestrado }\end{array}$ & 114 & $\begin{array}{c}\text { Amplatzer ASO } \\
12 \mathrm{~mm}\end{array}$ \\
\hline
\end{tabular}

CIV = comunicação interventricular; DVEVD = dupla via de entrada de ventrículo direito; DVEVE = dupla via de entrada de ventrículo esquerdo; DVSVD = dupla via de saída de ventrículo direito; EP = estenose pulmonar; PCA = persistência do canal arterial; SIV = septo interventricular; TGA = transposição dos grandes vasos .

TABELA 2

Dados demográficos do grupo 2

\begin{tabular}{|c|c|c|c|c|c|c|}
\hline & $\begin{array}{c}\text { Idade } \\
\text { (meses) }\end{array}$ & $\begin{array}{c}\text { Peso } \\
(\mathrm{kg})\end{array}$ & Diagnóstico & Conduto & $\begin{array}{l}\text { Tempo após } \\
\text { Fontan (meses) }\end{array}$ & Dispositivos \\
\hline 6 & 260 & 41 & $\begin{array}{c}\text { DVEVE/TGA } \\
\text { CIV/PCA }\end{array}$ & Intracardíaco & 86 & $\begin{array}{l}\text { P308/Balão } 15 \text { mm } \\
\text { (pré-dilatado) }\end{array}$ \\
\hline 7 & 128 & 10 & $\begin{array}{c}\text { DVEVE/ } \\
\text { TGA/CIV }\end{array}$ & Intracardíaco & 0,0 & $\begin{array}{l}\text { P308/Balão } 12 \text { mm } \\
\text { (pré-dilatado) }\end{array}$ \\
\hline 8 & 202 & 50 & $\begin{array}{c}\text { Atresia } \\
\text { tricúspide }\end{array}$ & Intracardíaco & 138 & $\begin{array}{l}\text { CP } 39 \text { mm/Balão } \\
20 \text { mm e } 23 \text { mm }\end{array}$ \\
\hline 9 & 56 & 11 & $\begin{array}{l}\text { Atresia tricúspide/ } \\
\text { concordância VA/EP }\end{array}$ & Dácron no 22 & 0,1 & $\begin{array}{r}\text { CP } 34 \text { mm/Balão } 15 \text { mm } \\
\text { (Hippocampus-Invatec } \\
6 \text { mm x } 24 \text { mm em APE e } \\
7 \text { mm x } 24 \text { mm em APD) }\end{array}$ \\
\hline 10 & 51 & 12 & $\begin{array}{c}\text { DSAV desbalanceado } \\
\text { com dominância direita } \\
\text { DVSVD }\end{array}$ & Dácron no 20 & 0,8 & $\begin{array}{c}\text { CP Covered } 39 \text { mm } \\
\text { Balão } 15 \text { mm }\end{array}$ \\
\hline 11 & 194 & 49,5 & $\begin{array}{c}\text { Isomerismo direito } \\
\text { DVEVENAV } \\
\text { comum/DVSVE/EP }\end{array}$ & $\begin{array}{l}\text { Homoenxerto } \\
\text { aórtico } 18 \mathrm{~mm}\end{array}$ & 123 & $\begin{array}{l}\text { CP Covered } 60 \text { mm } \\
\text { Balão } 16 \text { mm }\end{array}$ \\
\hline
\end{tabular}

APD = artéria pulmonar direita; $\mathrm{APE}$ = artéria pulmonar esquerda; CIV = comunicação interventricular; DSAV = defeito do septo atrioventricular; DVEVE = dupla via de entrada de ventrículo esquerdo; DVSVD = dupla via de saída de ventrículo direito; DVSVE = dupla via de saída de ventrículo esquerdo; $\mathrm{EP}=$ estenose pulmonar; $\mathrm{PCA}=$ persistência do canal arterial; TGA = transposição dos grandes vasos; $\mathrm{VA}=$ ventrículo arterial; $\mathrm{VAV}=$ válvula atrioventricular.

foram comparados com os dados obtidos previamente ao implante do stent ou dispositivo de Amplatzer ${ }^{\mathrm{TM}}$.

\section{Análise estatística}

Variáveis contínuas como idade, peso e dados pré e pós-intervenção são apresentadas como média e desvio padrão. A comparação das variáveis antes e após o implante dos dispositivos foi realizada com teste $t$ de Student para amostras pareadas. Valor de $\mathrm{P}<0,05$ foi considerado estatisticamente significante. Análise estatística foi realizada com o pacote estatístico SPSS versão 11.0 (SPSS Inc., Chicago, Estados Unidos). 
Manica JL, et al. Tratamento Percutâneo de Lesões Residuais e Complicações em Condutos de Pacientes Submetidos a Cirurgia de Circuito do Tipo Fontan. Rev Bras Cardiol Invasiva. 2010;18(4):435-42.

\section{RESULTADOS}

\section{Pacientes}

Os dados relativos aos dispositivos utilizados estão apresentados nas Tabelas 1 e 2 .

No grupo 1, a média de idade dos pacientes no momento do procedimento era de $174 \pm 53,5$ meses e o peso médio, de $30,7 \pm 6,8 \mathrm{~kg}$. Todos os procedimentos foram realizados de forma eletiva, em média $84,8 \pm 29,5$ meses após o procedimento.

No grupo 2, a média de idade dos pacientes no momento do procedimento era de 148,5 $\pm 84,6$ meses e o peso médio, de $28,9 \pm 19,8 \mathrm{~kg}$. Os 3 pacientes da amostra com menos de $15 \mathrm{~kg}$ realizaram o procedimento durante o período pós-operatório, ainda enquanto internados em Unidade de Cuidados Intensivos, por apresentarem sinais de congestão sistêmica e/ou baixo débito cardíaco refratários ao tratamento clínico. Outros 3 pacientes realizaram o procedimento de forma eletiva $115,7 \pm 26,7$ meses após o procedimento.

O paciente que recebeu simultaneamente ambos os dispositivos tinha 264 meses de idade no momento do procedimento e pesava $64 \mathrm{~kg}$. O procedimento foi realizado de forma eletiva 167 meses após a cirurgia de anastomose cavopulmonar total.

\section{Dados hemodinâmicos e angiográficos}

Entre os 5 pacientes do grupo 1 que receberam dispositivos para oclusão de fenestrações, a saturação média antes do procedimento foi de $82,6 \pm 7,5 \%$ para $90,4 \pm 7,5 \%$ logo após o procedimento $(P=0,001)$ (Figura 3). Nos 2 pacientes que receberam stents recobertos, a média do menor diâmetro do conduto antes do procedimento era de 15,5 $\pm 0,7 \mathrm{~mm}$, passando para $17,9 \pm 1,2 \mathrm{~mm}$ após o procedimento. Um paciente que recebeu stent recoberto apresentava, à angiografia de controle imediatamente após o implante, shunt residual através da fenestração. Os pacientes submetidos a implante de prótese de Amplatzer ${ }^{T M}$ não apresentaram, à angiografia de controle, shunt residual através da prótese. A duração média do procedimento foi de $114 \pm 72,4$ minutos. Não foram observadas complicações relacionadas ao procedimento.

Entre os 6 pacientes do grupo 2 que receberam dispositivos percutâneos para alívio da obstrução dos condutos intra ou extracardíacos, a saturação média antes do procedimento foi de $81,8 \pm 8,9 \%$ para $91,3 \pm$ $8,7 \%$ logo após o procedimento $(P=0,01)$ (Figura 3). Já o menor diâmetro do conduto passou de uma média de $6,9 \pm 4,8 \mathrm{~mm}$ antes do procedimento para uma média de 16,6 $\pm 3,5 \mathrm{~mm}$ após o procedimento $(P=0,02)$ (Figura 4). A duração média do procedimento foi de $78,3 \pm 17,5$ minutos. Não foram observadas complicações relacionadas ao procedimento.

O paciente que realizou ambos os procedimentos apresentou ganho de aproximadamente 30\% em relação ao menor diâmetro do conduto, passando de 11,7 mm para $16 \mathrm{~mm}$ de diâmetro. Foi também observada expressiva melhora da saturação sistêmica, passando de $60 \%$ antes do procedimento para $90 \%$ após o alívio da obstrução e oclusão da fenestração, demonstrando o significativo benefício do procedimento. O procedimento teve duração de 90 minutos e não foram observadas complicações.

\section{Seguimento}

Os pacientes do grupo 1, submetidos a oclusão percutânea de fenestração cirúrgica, tiveram seguimento médio de 17 meses. Desses 5 pacientes, 3 se apresentaram assintomáticos na última consulta, 1 paciente apresentava dessaturação arterial persistente compatível com shunt residual após o procedimento e 1 paciente, HIV positivo, faleceu 36 meses após o procedimento por sepse. Nenhum paciente apresentou sinais de obstrução do conduto durante o seguimento.

Os pacientes do grupo 2, submetidos a dilatação de conduto cavopulmonar com stent, tiveram seguimento médio de 35 meses. Desses 6 pacientes, 3 se apresen-

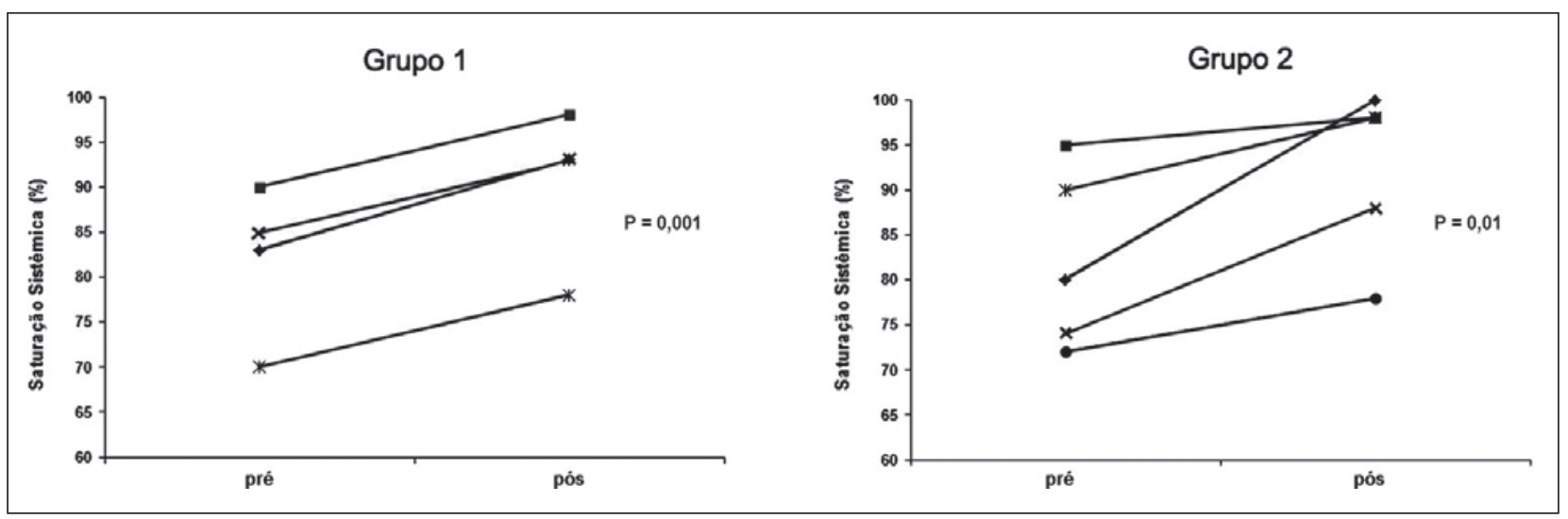

Figura 3 - Comparação entre a variação da saturação sistêmica antes e após o procedimento percutâneo nos grupos 1 e 2 . 
Manica JL, et al. Tratamento Percutâneo de Lesões Residuais e Complicações em Condutos de Pacientes Submetidos a Cirurgia de Circuito do Tipo Fontan. Rev Bras Cardiol Invasiva. 2010;18(4):435-42.

taram assintomáticos na última consulta, inclusive a paciente submetida a implante de stent recoberto no

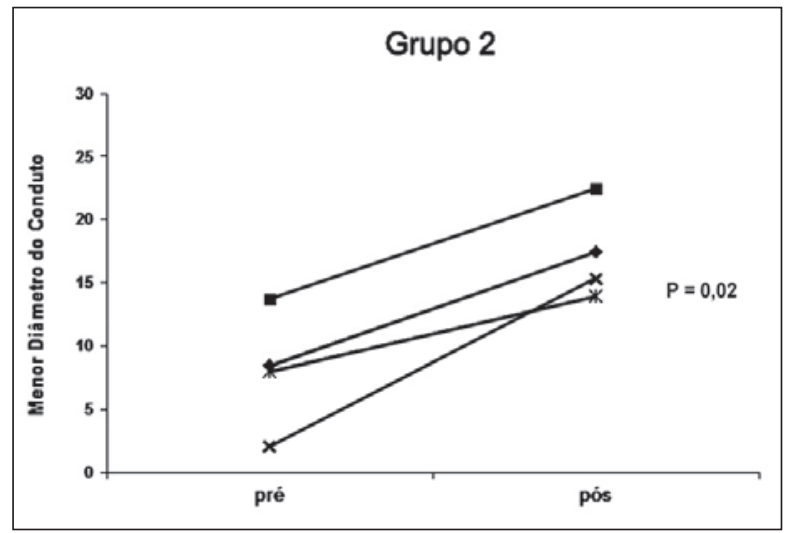

Figura 4 - Comparação do menor diâmetro do conduto antes e após o procedimento percutâneo no grupo 2 . pós-operatório por trombose maciça do conduto (paciente 10), e 1 paciente apresentava queixa de cansaço aos médios esforços, porém em nenhum desses pacientes foram observados sinais de congestão sistêmica ou obstrução do conduto. O único paciente do estudo que faleceu ainda durante a internação hospitalar (paciente 9) foi submetido a "reconstrução" da anastomose cavopulmonar com implante de stents no conduto e em ambas as artérias pulmonares no pós-operatório imediato de cirurgia de Fontan em estado grave (Figura 5). Esse paciente faleceu 5 dias após o procedimento por choque cardiogênico. Outro paciente faleceu 10 meses após o procedimento por infecção respiratória, provavelmente relacionada ao vírus da gripe A. Nesse momento o paciente se encontrava assintomático, a despeito da extensa rede de colaterais venoatriais observada previamente ao alívio da obstrução com stent recoberto.

O paciente que foi submetido a implante simultâneo de oclusor septal e stent não-recoberto se encontra-
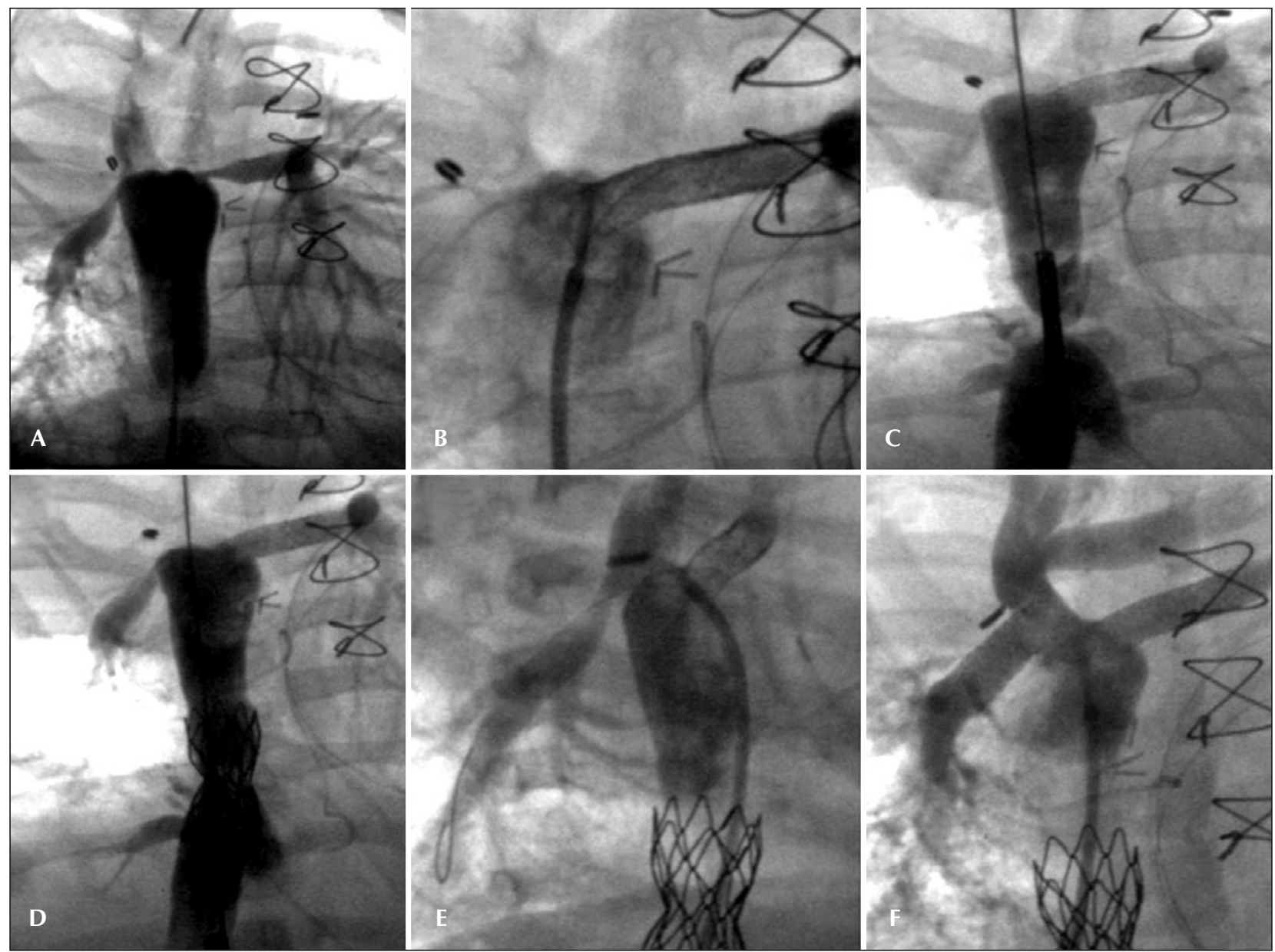

Figura 5 - "Reconstrução" de anastomose cavopulmonar total com tubo de dácron em pós-operatório imediato. Em A, angiografia no conduto demonstrando estenose na anastomose distal do conduto acometendo a origem de ambas as artérias pulmonares. Em B, implante de stent na artéria pulmonar esquerda. Em C, angiografia demonstrando estenose grave na anastomose proximal do conduto. Em D, implante de stent na anastomose proximal do conduto. Em E, angiografia demonstrando estenose na origem do ramo pulmonar direito. Em $\mathrm{F}$, aspecto final de ambos os ramos pulmonares. 
Manica JL, et al. Tratamento Percutâneo de Lesões Residuais e Complicações em Condutos de Pacientes Submetidos a Cirurgia de Circuito do Tipo Fontan. Rev Bras Cardiol Invasiva. 2010;18(4):435-42.

va assintomático e acianótico na última consulta, realizada 9 meses após o procedimento.

\section{DISCUSSÃO}

Desde a introdução da cirurgia de Fontan, em 1971, ${ }^{1}$ várias modificações técnicas foram acrescentadas com o intuito de melhorar a sobrevida dos pacientes e diminuir a incidência de complicações relacionadas ao procedimento. A longevidade desses condutos, tanto intra como extracardíacos, é um aspecto a ser considerado no momento da escolha da opção cirúrgica, bem como a realização ou não de fenestrações cirúrgicas. Essa decisão poderá ter grande impacto na necessidade de procedimentos adicionais futuros.

Diversos estudos demonstraram que a utilização de fenestrações, seja em condutos intra ou extracardíacos, reduz a pressão venosa sistêmica no período pós-operatório imediato, melhora o débito cardíaco e diminui a morbidade e a mortalidade cirúrgica desse procedimento, principalmente em pacientes de alto risco.$^{6,8}$ Entretanto, a persistência de fluxo direita-esquerda através desses defeitos provoca cianose, risco aumentado de embolia sistêmica, diminuição da tolerância aos esforços e necessidade de novo procedimento percutâneo para o fechamento da fenestração. ${ }^{9}$

Vários estudos demonstraram a eficácia e a segurança do fechamento eletivo de fenestrações cirúrgicas por via percutânea com diversos dispositivos diferentes, com destaque para os oclusores septais e os stents recobertos. ${ }^{4,5,10-12} \mathrm{~A}$ utilização de stents recobertos possui a vantagem de não expor material metálico na cavidade atrial pulmonar e de permitir realizar, simultaneamente, o tratamento de estenoses associadas. Nesses casos, os stents cobertos aumentam a segurança do procedimento na presença de condutos calcificados, podendo tratar os efeitos desastrosos de eventual ruptura. Essas próteses ainda são ideais na oclusão de fenestrações de difícil delimitação anatômica, com trajeto tortuoso ou múltiplas. ${ }^{13}$ Entretanto, a necessidade de bainhas de maior perfil pode dificultar ou até mesmo impedir a realização do procedimento em crianças menores, de baixo peso. O presente estudo relata a efetividade do procedimento de oclusão percutânea de fenestrações com bons resultados em relação à melhora da saturação sistêmica em todos os pacientes, independentemente do dispositivo utilizado, com apenas um paciente apresentando shunt residual com queda da saturação no seguimento a curto prazo. Além disso, a ausência de complicações demonstra a segurança do procedimento, assim como já havia sido descrito na literatura.

Além de raro nas grandes séries ${ }^{2,14}$, o desenvolvimento de obstrução dos condutos, seja por calcificação, trombose ou distorção após crescimento somático dos pacientes, pode ser tratado por via percutânea. A dilatação com balão de condutos de pacientes submetidos a anastomose cavopulmonar total mostrou-se pouco efetiva nos raros casos em que foi descrita; ${ }^{15,16}$ entretanto, assim como nos casos de condutos entre o ventrículo direito e a artéria pulmonar, o implante de stents em artérias pulmonares em pacientes em pósoperatório de cirurgia de Fontan tem resultados satisfatórios. ${ }^{17,18}$ Por outro lado, poucos estudos relatam o uso desses dispositivos para o alívio de estenoses de condutos de anastomose cavopulmonar total. ${ }^{7,10}$

O presente estudo demonstrou a efetividade do implante de stents em condutos intra e extracardíacos tanto em pacientes na fase aguda de pós-operatório como quando realizado em decorrência de obstrução crônica dos condutos. A necessidade de implante de stents recobertos nesse espectro de pacientes é discutível, e neste estudo o procedimento foi realizado em dois casos por razões distintas. Em um caso de obstrução aguda de conduto por trombose maciça ainda no período pós-operatório, a utilização de stent recoberto se justificou principalmente pelo risco de desprendimento dos trombos e consequente embolização para a árvore pulmonar (paciente 10). Já no outro paciente, foi utilizado dispositivo recoberto por se tratar de homoenxerto calcificado com possível risco de ruptura. Nesse paciente, que apresentava extensa rede de colaterais intra-hepáticas para o átrio direito, observou-se significativo aumento do fluxo anterógrado através do conduto após o implante do stent em detrimento do fluxo desviado pelas colaterais venoatriais, demonstrando a efetividade da técnica. Cabe lembrar que significativo aumento da saturação sistêmica foi observado em todos os pacientes submetidos a alívio de estenoses de condutos sem evidência de fenestrações, o que provavelmente está relacionado a melhora do débito venoso sistêmico, mesmo no caso de pacientes sem a presença de colaterais aparentes. É importante ressaltar que o único caso de óbito precoce ocorreu no período pós-procedimento imediato em paciente submetido a "reconstrução" percutânea da anastomose cavopulmonar com implante de stents em conduto e em ambas as artérias pulmonares (paciente 9). Neste estudo foram observadas duas mortes tardias não relacionadas ao procedimento: uma por sepse em paciente HIV positivo 36 meses após o procedimento (paciente 5), e outra por suspeita de gripe A 10 meses após o procedimento (paciente 11). O seguimento dos pacientes parece revelar que os resultados são satisfatórios pelo menos a médio prazo, sem necessidade de reintervenção na pequena amostra relatada. Apenas um paciente apresentava fluxo residual após fechamento de leak de homoenxerto aórtico com stent recoberto com persistência da cianose durante acompanhamento ambulatorial. Esse paciente deverá ser submetido a novo procedimento percutâneo no futuro para tentativa de oclusão do fluxo residual e melhora da saturação sistêmica (paciente 1).

Deve-se ressaltar a possibilidade de realização de ambos os procedimentos simultaneamente por meio de duas abordagens diferentes. No presente estudo, no mesmo procedimento foi implantado stent não- 
Manica JL, et al. Tratamento Percutâneo de Lesões Residuais e Complicações em Condutos de Pacientes Submetidos a Cirurgia de Circuito do Tipo Fontan. Rev Bras Cardiol Invasiva. 2010;18(4):435-42.

recoberto para alívio de obstrução de conduto cavopulmonar e, posteriormente, foi implantada prótese de Amplatzer ${ }^{\mathrm{TM}}$ para oclusão da fenestração. O procedimento ocorreu sem maiores complicações, podendo ser realizado de maneira rotineira, principalmente nos casos com fenestrações distantes do local da obstrução. Por outro lado, a possibilidade de alívio de obstrução de conduto e oclusão de fenestração apenas com o implante de stent recoberto deve ser lembrada em casos com defeitos próximos e fenestrações de difícil localização, conforme já descrito na literatura. ${ }^{4,10}$

\section{CONCLUSÃO}

Apesar do número restrito de pacientes, este estudo representa uma população de pacientes submetidos previamente a diversas técnicas de cirurgia de anastomose cavopulmonar total que se beneficiam da terapêutica percutânea em diferentes momentos de sua evolução, por diferentes motivos. O implante percutâneo de stents em condutos de Fontan, tanto intra como extracardíacos, mostrou-se efetivo e seguro nos pacientes com evolução crônica de obstrução dos condutos. Da mesma forma, deve-se salientar que, em pacientes com evolução extremamente desfavorável no período pós-operatório imediato, com sinais de congestão sistêmica refratária à terapêutica clínica, o mesmo procedimento pode ser a única alternativa capaz de manter o circuito pérvio e de preservar a circulação no circuito de Fontan. Além disso, a oclusão percutânea eletiva de fenestrações cirúrgicas também se mostrou efetiva, com pequeno índice de fluxo residual e meIhora da saturação sistêmica persistente durante o seguimento ambulatorial, demonstrando o papel fundamental do hemodinamicista no tratamento a longo prazo de pacientes com cardiopatias complexas não passíveis de reparo biventricular.

\section{CONFLITO DE INTERESSES}

Os autores declararam inexistência de conflito de interesses relacionado a este manuscrito.

\section{REFERÊNCIAS}

1. Fontan F, Baudet E. Surgical repair of tricuspid atresia. Thorax. 1971;26(3):240-8.

2. Giannico S, Hammad F, Amodeo A, Michielon G, Drago F, Turchetta A, et al. Clinical outcome of 193 extracardiac Fontan patients: the first 15 years. J Am Coll Cardiol. 2006; 47(10):2065-73.

3. Lemler MS, Scott WA, Leonard SR, Stromberg D, Ramaciotti C. Fenestration improves clinical outcome of the Fontan procedure: a prospective, randomized study. Circulation. 2002;105(2):207-12.
4. Marini D, Boudjemline Y, Agnoletti G. Closure of extracardiac Fontan fenestration by using the covered Cheatham Platinum stent. Catheter Cardiovasc Interv. 2007;69(7):1002-6.

5. Pihkala J, Yazaki S, Mehta R, Lee KJ, Chaturvedi R, McCrindle BW, et al. Feasibility and clinical impact of transcatheter closure of interatrial communications after a fenestrated Fontan procedure: medium-term outcomes. Catheter Cardiovasc Interv. 2007;69(7):1007-14.

6. Bridges ND, Lock JE, Castaneda AR. Baffle fenestration with subsequent transcatheter closure. Modification of the Fontan operation for patients at increased risk. Circulation. 1990; 82(5):1681-9.

7. Ewert P, Schubert S, Peters B, Abdul-Khaliq H, Nagdyman $\mathrm{N}$, Lange PE. The CP stent-short, long, covered-for the treatment of aortic coarctation, stenosis of pulmonary arteries and caval veins, and Fontan anastomosis in children and adults: an evaluation of 60 stents in 53 patients. Heart. 2005; 91(7):948-53.

8. Gentles TL, Mayer JE Jr, Gauvreau K, Newburger JW, Lock $J E$, Kupferschmid JP, et al. Fontan operation in five hundred consecutive patients: factors influencing early and late outcome. J Thorac Cardiovasc Surg. 1997;114(3):376-91.

9. Quinones JA, Deleon SY, Bell TJ, Cetta F, Moffa SM, Freeman JE, et al. Fenestrated Fontan procedure: evolution of technique and occurrence of paradoxical embolism. Pediatr Cardiol. 1997;18(3):218-21.

10. Hijazi ZM, Ruiz CE, Patel H, Cao QL, Dorros G. Catheter therapy for Fontan baffle obstruction and leak, using an endovascular covered stent. Cathet Cardiovasc Diagn. 1998; 45(2):158-61.

11. Gamillscheg A, Beitzke A, Stein J, Rupitz M, Zobel G, Rigler B. Transcatheter coil occlusion of residual interatrial communications after Fontan procedure. Heart. 1998;80(1):49-53.

12. Goff DA, Blume ED, Gauvreau K, Mayer JE, Lock JE, Jenkins KJ. Clinical outcome of fenestrated Fontan patients after closure: the first 10 years. Circulation. 2000;102(17):2094-9.

13. Rothman A, Evans WN, Mayman GA. Percutaneous fenestration closure with problematic residual native atrial septum. Catheter Cardiovasc Interv. 2005;66(2):286-90.

14. Kim SJ, Kim WH, Lim HG, Lee JY. Outcome of 200 patients after an extracardiac Fontan procedure. J Thorac Cardiovasc Surg. 2008;136(1):108-16.

15. Sohn S, Kashani IA, Rothman A. Partial and transient relief of conduit obstruction by low-pressure balloon dilation in patients with congenital heart disease. Cathet Cardiovasc Diagn. 1995;34(1):35-40.

16. Lloyd TR, Marvin WJ Jr, Mahoney LT, Lauer RM. Balloon dilation valvuloplasty of bioprosthetic valves in extracardiac conduits. Am Heart J. 1987;114(2):268-74.

17. Bhole V, Wright JG, De Giovanni JV, Dhillon R, Miller PA, Desai T, et al. Transcatheter interventions in the early postoperative period after the Fontan procedure. Catheter Cardiovasc Interv. 2010 Jun 1. [Epub ahead of print].

18. Rossi RI, Manica JL, Borges MS, Machado P. Implante de stent não-valvado na via de saída do ventrículo direito: forma simples e efetiva de retardar nova intervenção cirúrgica. Rev Bras Cardiol Invasiva. 2009;17(1):102-9. 